\title{
Beschreibung eines sehr kleinen menschlichen Eies.
}

\author{
Von \\ Prof. Friedrich Ahlfeld.
}

(Mit Abbildungen Tafel VIX.)

Am 26. Juni 1875 bekam ich ein sehr kleines menschliches Ei zur Untersuchung, das der Grösse nach an das von Reichert beschriebene erinnert. Leider hatte ich das Unglück, bei der Compression des Eies den Inhalt entschlüpfen zu lassen und nur die Eihüllen zu behalten. Da mir aber auch das Nest des Eies mit übergeben worden war und an diesem sowohl wie an den Eihäuten selbst mancherlei der Untersuchung werth erschien, so nahm ich dieselbe vor. Dass ich jetzt erst zur Veröffentlichung derselben schreite, geschah, weil ich wiederholt später Eier aus sehr früher Zeit der Schwangerschaft zu untersuchen Gelegenheit hatte, die ich mit dem obigen vergleichen konnte.

Das Eichen stammte von einer verheiratheten Frau, die bereits ein Mal geboren. Frau F. pflegte genau den Eintritt der Menstruation zu notiren, und ich erfuhr, dass die letzten Perioden eintraten am 11. December Abends, am 13. Januar 1875 Nachmittags, am 11. Februar Vormittags, am 11. März Vormittags, am 10. April Nachmittags, am 11. Mai Vormittags. Die am 10. oder 11. Juni erwartete Menstruation blieb aus, ohne dass bisher eine subjective Störung auf Schwangerschaft hinwies. Am 25. Juni erfolgte eine Blutung, deren halber ich zu Rathe gezogen wurde. Ich machte die Frau darauf aufmerksam, dass vielleicht ein kleines Ei abgehen könnte und bat sie, jeden ihr auffallenden Blutklumpen mir aufzubewahren. Am 26. Juni Vormittags, kurz ehe 
ich meinen Besuch machte, war ein kleiner Blutklumpen abgegangen, den Frau F. in Wasser gelegt hatte. Es war Uterusschleimhaut, an der man sofort eine Hervorragung sah, die der Reflexa entsprach. Die weitere Untersuchung wurde nun auf meinem Arbeitszimmer vorgenommen.

Es zeigten sich zwei grössere Lappen der Uterinschleimhaut, die den beiden Hälften einer Uterushöhle entsprachen, aber nicht, wie sonst so häufig, die Gestalt der Uterushöhle genau wiedergaben. Auf dem einen Lappen fand sich eine erbsengrosse Erhabenheit. Dieselbe wurde unter Wasser geöffnet, und es präsentirten sich kleine Chorionzotten. Die Reflexa liess sich sehr loicht von den Zottenspitzen entfernen, so dass man den Eindruck einer nur sehr oberflächlichen Verbindung beider mit einander hatte. Ebensowenig machte es Schwierigkeiten, das kleine Ei von der Serotina abzuheben.

Das Ei war nahezu rund und zeigte einen sehr spärlichen Besatz von Zotten. Die letzteren waren sehr kurz; ich werde ihre Länge weiter unten genauer angeben. Mit dem Auge und mit der Loupe schien der Inhalt ganz gleichmässig zu sein. Ich entdeckte keinen geformten Theil. Um mich über den Inhalt zu vergewissern, legte ich das Ei zwischen zwei Objectträger, um eine Vergrösserung des Mikroskops benutzen zu können. Bei dieser Manipulation zerplatzte das Ei und es floss eine schleimige Masse heraus, in der ich im Augenblick des Herausfliessens keinen festen Theil wahrnehmen konnte. Es blieben mir nur die Eihüllen, die ich im Weiteren beschreiben werde. Ich färbte die übriggebliebenen Theile mit Silbersalpeter und bei einer späteren Untersuchung mit Hämatoxilin.

Die Umhüllungshaut des Eichens besteht aus einer zarten epithelialen Membran. Das Epithel ist grosskernig, so dass nur immer ein kleiner Protoplasmastreifen zwischen den einzelnen Kernen zu bemerken ist. Das Protoplasma ist dunkelkörnig; es lassen sich die Zellgrenzen nur sehr undeutlich nachweisen. An einzelnen Stellen sieht man im Protoplasma Vacuolen, die mit Hartnack 7 sehr deutlich zu bemerken sind. Das Epithel ist überall gleichmässig fein granulirt, so dass man auch einzelne kleine Partien sofort von dem zweiten Gewebe, der inneren Haut unterscheiden kann. Ausserdem hat die doppelte Färbung des Präparates die beiden Schichten deutlich unterschieden. Durch das Silber ist das Epithel ziemlich dunkel gefärbt worden, wäh- 
rend die weiter zu beschreibende Bindegewebslage wenig annahm, hingegen die Färbung durch Haematoxilin um so intensiver behielt.

Diese letztere Hülle liegt der Epithelhülle allseitig gleichmässig an. Sie besteht aus einem zarten Bindegewebe mit auffallend langen, zum Theil merkwürdig geschlängelten, gebogenen, schmalen Zellen. Dieselben liegen bald weiter auseinander, bald näher, überall aber nehmen sie die verschiedensten Richtungen ein, kreuzen sich, laufen parallel u. s. w. Die Intercellularsubstanz zeigt ein feines maschiges Bild.

Die Zotten sind im Maximum circa $10 \mathrm{Mm}$. lang. Es sind nur Theilungen bis zum zweiten Grade vorhanden. Sie stehen ziemlich verreinzelt, doch habe ich vergessen mir zu notiren, ob eine regelmässige Anordnung derselben zu bemerken war. Zur Zeit, wo das Ei zerstückelt unter einem Deckgläschen liegt, ist dies nicht mehr nachweisbar. Die Zotten werden von demselben Epithel bedeckt, welches dem Eie als Umhüllungshaut dient, nur sind die Epithelien auf denselben stellenweise kleiner als auf der Eioberfläche. Die kleinsten Zotten und Zottensprossen bestehen nur aus Epithel. Zumeist haben sie einen dünnen Stiel und ein kolbiges Ende. Im letzteren scheint sich zuerst ein Hohlraum zu bilden. Man bemerkt an einigen Präparaten in der Mitte der kolbigen Anschwellung eine Vacuola. Sind die Zotten durchweg hohl, so sieht man in ihrem Innern einen Bindegewebsstock, der aus der zweiten oben beschriebenen Eihülle stammt. Nicht immer liegt dieses Bindegewebe dem Epithel dicht an, sondern zwischen beiden befindet sich ein Zwischenraum. Auch sieht man an vielen Stellen, dass das Epithel sich von dem Zottenstocke entfernt hat. Eine Verbindung beider konnte daher noch nicht vorhanden sein. Es ist aus dieser Thatsache zu schliessen, dass durch selbständiges Wachsthum des Epithelmantels die Zotte sich stetig vergrössert und dass erst in die hohle Zotte hinein das Bindegewebsstratum wächst.

Das Nest, bestehend aus der Decidua serotina, einer kleinen Partie der im Umkreise anhängenden Decidua vera und aus der vollständig erhaltenen, nur durch einen Längsschnitt (zur Herausnahme des Eies gemachten) gespaltenen Decidua reflexa, legte ich in Spiritus und härtete es. Auch jetzt, nach 2\% Jahren, ist die Schleimhaut sehr weich geblieben und schneidet sich in Folge dessen nicht gerade leicht.

Ich beschreibe einen Schnitt, der durch die Mitte des Nestes 
(also im Durchmesser) geführt, durch sämmtliche Partien hindurchgeht. Legt man die beiden freien Enden der Reflexa wieder aneinander, so beträgt am Spirituspräparate der Raum, in dem das Ei gelegen, im Durchmesser 3,3 Mm. Der Raum ist daher geschrumpft. Die Serotina beträgt an ihrer stärksten Stelle 2,1 Mm., die grösste Längsausdehnung, von einer Umschlagstelle zur anderen, misst 6,0 Mm. Die Reflexa wird auf der einen Seite des Sagittalschnittes von einem dickeren Gewebe gebildet, während die andere Hälfte aus einem dünnen fadenförmigen Strange besteht.

Die Serotina zeigt im Ganzen das in der letzten Zeit öfter beschriebene Bild der aufgelockerten wuchernden Schleimhaut. Nur in der Mitte des Präparates sieht man einen dunkleren Knoten, der bei mikroskopischer Untersuchung sich als fibrinösdegenerirter Bluterguss documentirt. Vergleicht man Schnitte vom Rande des Nestes, so fehlt hier der Bluterguss. Er liegt also nur im Centraltheile des Nestes.

Der dickere Theil der Decidua reflexa ähnelt im Ganzen der Decidua serotina resp. vera, nur bemerkt man, dass die compacte Zellenschicht der Decidua in stärkerem Maasse an ihm gewuchert ist als die Drüsenschicht. Der längere Theil der Reflexa, das fadenförmige Stïck, zeigt eine Zusammensetzung, wie ich sie in den mir bekannten Fällen bisher nicht gefunden habe. An der Umschlagsstelle sieht man, wie von der Seite der Serotina, wie auch von Seite der Vera, je eine compacte Schicht und die zwischen beiden liegende gemeinsame Drüsenschicht sich erheben und in die Reflexa übergehen. Weiter von der Umschlagsstelle entfernt, wird die Zwischendrüsenschicht immer schmaler und schmaler, bis zuletzt die Reflexa nur noch aus den dicht aneinander getretenen beiden compacten Zellenschichten besteht und gar kein Zwischenraum mehr zu bemerken ist. Auch in der Reflexa findet man Blutergüsse, theils ältere, sich organisirende, theils frischere.

Die Vera der Gebärmutter, also die Theile der Schleimhaut, die nicht zum Neste gehören, zeigt keine Abweichungen. Sie bietet ein Bild, wie wir es in Langhans and Leopold's Arbeiten beschrieben und zum Theil abgebildet finden. Nur in einem Punkte finde ich Unterschiede: die in der Drüsenschicht noch bemerkbaren Epithelien beschränken sich auf minimale Partien, während in den Zeichnungen Leopold's auch in den inneren 
Theilen der Drüsenschicht zahlreiche Stellen mit noch erhaltenen Epithelien gezeichnet and beschrieben werden. Es ist wohl denkbar, dass dieser Unterschied zwischen Deciduen, die aus der Leiche herausgenommen, und solchen, die ausgestossen wurden, in dem Zerfalle der Gewebe seinen Grund hat, der mit dem Aborte eimherzugehen pflegt.

Schon bei der Eröffnung der Reflexa konnte ich mich bei dem Anblicke der ziemlich vereinzelt stehenden Chorionzotten des Gedankens nicht erwehren, dass das beschriebene Ei ein unregelmässig entwickeltes sei. Es bestätigte sich die Annahme noch durch das Auffinden eines das Centrum der Serotina fïllenden Blutergusses. Daher nahm ich auch an, als ich keine Fötusanlage wahrnahm, dass dieselbe ebenfalls, wie so häufig in frühzeitig abgegangenen Eiern, zu Grunde gegangen. Doch ist die Möglichkeit nicht auszuschliessen, dass, ähnlich wie im Reichert'schen Eie, auch im unserigen der gallertartige Inhalt den Embryo noch in einem sehr frïhen Zustande der Entwickelung enthalten habe. Dafür spricht vor Allem der Mangel einer dritten Haut, die als Amnion aufzufassen wäre. Wäre dieselbe vorhanden gewesen, sie könnte mir nicht entgangen sein. Doch wäre auch hier die Möglichkeit vorhanden, dass Embryo und Amnion zerfallen gewesen sei. Dass das Ei einer sehr zeitigen Periode entstammt, beweisen die Zotten, welche in der Mehrzahl noch ungetheilt, in einer kleineren Anzahl ein Mal getheilt und nur in einzelnen Exemplaren eine beginnende zweite Sprossung zeigen. In Reichert's Eie waren die längsten Zotten 0,2 $\mathrm{Mm}$. lang. Ein Theil derselben trug Nebenästchen. Die Zotten zeigen an ihren Endkuppen keine Verletzungen ihres Epithels. Es ist daher anzunehmen, dass ihre Verbindung mit der Uterusschleimhaut nur eine sehr oberflächliche war. Bestimmt kann man folgern, dass sie nicht in die Drüsen eingewachsen gewesen sind. Man würde dann nothwendigerweise Verletzungen des Zotten- oder des Drüsenepithels wahrnehmen müssen, welches in letzterem Falle der Zottenkuppe anhaften müsste.

Zahlreiche Aborte aus den ersten zwei Monaten der Schwangerschaft, die mir zu Händen kamen, lassen ausser Zweifel, dass die Ansicht von. Braxton Hicks') und Langhans²),

1) Obstetrical Transactions, XIV, p. 149.

2) Archiv für Anatomie und Physiologie 1877, 2. u. 3. Heft, S. 231. 
wonach das $\mathrm{Ei}$ nur sehr oberflächlich der Schleimhaut anklebt, nicht mit seinen Zotten in die Drüsenlumina hineingewuchert sei, die richtige ist. Man findet die Zottenkuppen auch bei Eiern aus der 6., 7. Woche der Schwangerschaft noch vollständig intact und die anhängenden Theile der Decidua reflexa nur oberflächlich an den Zotten befestigt. Nur wenn Blutergüsse die Decidua zerstört haben, was freilich in einer grossen Zahl der Aborte der Fall ist, sind die Chorionzotten in das umliegende Gewebe eingebettet.

Einige in letzter Zeit mehrfach discutirte Fragen im Betreff der histologischen Zusammensetzung sehr frühzeitiger menschlicher Eier, kamn ich am besten besprechen, wenn ich die beiden im letzten Jahre erschienenen Beschreibungen von Breus (Wiener medicinische Wochenschrift 1877, S. 502) und von Beigel und Löwe (dieses Archiv, Bd. XII, S. 421) mit meinen Befunden vergleiche.

Mit der Beschreibung, die Breus giebt, stimmen meine Präparate in auffallender Weise überein. Wenn er Seite 504 schreibt: „Man konnte nämlich an dieser (Umbüllungsmembran mit Zotten) eine äussere epitheliale Lamelle von einer inneren, aus unreifem Bindegewebe bestehenden Schichte abgrenzen. Ebenso an den Zotten, von welchen sich sogar Bilder fanden, wo eine dem sogenannten Mantelgewebe der Chorionzotten entsprechende, reichliche und lange Epithelsprossen tragende äussere Schichte von der bindegewebigen Axe derselben theilweise zurückgestreift war," so ist dies genau dasselbe, was auch ich an verschiedenen Stellen gesehen habe. In meinem Falle lassen sich Gefässe in den Zottền nicht mit Bestimmtheit nachweisen. Es finden sich wohl stellenweise in Doppelreihen angeordnete Zellen; doch möchte ich nicht entscheiden, ob diese nicht, durch Faltung des Bindegewebes, Kunstproducte sind. Im Innern der Bindegewebsschicht hat Breus noch einen faltig-lamellösen Belag gefunden, den er für ein Gerinnungsproduct hält. So wenigstens verstehe ich die Beschreibung bei Breus, während Beigel und Löwe (S. 430) annehmen, Breus habe die innere Eihaut, wie auch Reichert, für ein Gerinnungsproduct (Alkoholwirkung) erklärt. Zur Breus'schen Beschreibung passt auch dessen Annahme, dass eine kleine Protuberanz an der inneren Fläche der Eihaut der in Alkohol geschrumpfte Embryo sei.

In der Arbeit von Beigel und Löwe finden wir Befunde und Hypothesen, die von den jetzt geläufigen sich bedeutend 
unterscheiden. Leider ist die Beschreibung des Eies nicht mit der nöthigen Genauigkeit gemacht worden, um ein klares Bild zu. erlangen. Auch die kritische Besprechung, der in der Literatur bereits veröffentlichten Beschreibungen frühzeitiger menschlicher Eier entbehrt der nöthigen Genauigkeit; wenigstens muss ich dies von dem Referate der Breus'schen Arbeit annehmen, indem bei Breus thatsächlich zum Theil etwas ganz anderes zu lesen ist, als dies Beigel und $\mathrm{L}$ öwe gethan haben. $\mathrm{Zu}$ verwundern ist nur, dass Breus nicht selbst die verschiedenen Missverständnisse aufgeklärt hat, als er in der Wiener medicinischen Wochenschrift (1878, Nr. 17) ein Referat über Beigel's und Löwe's Aufsatz gegeben hat.

Ueber das Alter des Beigel'schen Eies bleibt man im Unklaren. Die Angabe, dass zwei Monate nach einem Aborte die Ausstossung stattgefunden, wird hinfällig, da die Autoren selbst angeben, dass zwei Monate nach einem Abortus Blutungen eingetreten seien, die sich in unregelmässigen Zeiträumen einstellten, und erst bei der letzten dieser.Blutungen sei die Geburt des Eies erfolgt. Also wahrscheinlich nach einem grösseren Zwischenraume als nach zwei Monaten.

Auf die Beigel-Löwe'sche Beschreibung näher einzugehen, ist den Lesern dieses Archivs gegenüber überflüssig, da sie ohne grosse Mühe den kurzen Aufsatz durchlesen können. Treffend charakterisirt Breus die Arbeit, wenn er in seinem Referate sagt: „Entsprechend diesen unklaren makroskopischen Verhältnissen des Gebildes gestalten sich auch die mikroskopischen."

Wenn ich nur die Abbildungen ohne Text vor mir gehabt hätte, so würde ich das Ei für ein solches gehalten haben, in dem der Fötus zerfallen. Die kleinen Bläschen wären dann als Dotterblase, blasenförmige Anhänge des Amnion, wie solche nicht selten vorkommen; u. s. w. aufzufassen gewesen. Die grosse Blase, „das eigentliche $\mathrm{Ei}^{\circ}$, würde dann das Amnion dargestellt haben. Jedenfalls ist das Ei im Zustande der Fäulniss. Es beweist dies die Abbildung des Zottenepithels wie auch die Beschreibung der subepithelialen Bindegewebsschicht.

Wenn die Autoren Reichert den Vorwurf machen, er habe die Zotten der Tunica externa ,unrichtig“ gezeichnet, insofern er sie als Hohlgebilde darstellte, so stehen sie mit dieser Behauptung allein. Auch an dem von mir beschriebenen Eie zeigten die Zotten eine Höhlung, sobald sie über eine bestimmte Grösse Archiv f. Gynäkologie. Bd. XrI. Hft. 2. 
248 Ahlfeld, Beschreibung eines sehr kleinen menschlichen Fies.

hinaus sich entwickelt haben, wie ich dies oben genauer beschrieben habe.

Die Annahme der beiden Autoren, dass möglicherweise die Umhüllungshaut mit den Zotten ein Product des Genitaltractus, dem Eie also erst während seiner Wanderung zu Theil werde, die Annahme ferner, dass die kleinen Bläschen selbst die Eier seien, also in diesem Falle mehrere abortive Eier in der Zottenhülle sich befänden, halte ich bis jetzt nicht für discutirbar, da die Autoren gar keine Gründe nennen, denen zu Folge die bisher geltende Ansicht fallen gelassen werden muss.

In allen bisher beschriebenen Beobachtungen früheitiger menschlicher Eier lässt sich die epitheliale Umbüllungshaut mit Kölliker als Serosa auffassen, die derselben innen angelegte Bindegewebsmembran wird ron der Allantois producirt sein, wiedenn wohl anzunehmen ist, dass, vielleicht mit Ausnahme des Eies von Reichert, in allen übrigen Fällen der bereits angelegte Fötus wieder zu Grunde gegangen ist.

\section{Erklärung der Abbildungen.}

Figur 1. Halbschematische Darstellung des beschriebenen Eies innerhalb des Einestes.

Figur 2. Epithelhülle, dem Bindegewebsstratum aufgelagert.

Figur 3. Zotte mit Epithelmantel und Bindegewebsstock.

F.igur 4. Beginn der Zottenbildung aus der epithelialen Hülle. Im Innern der Zotte drei Vacuolen. 

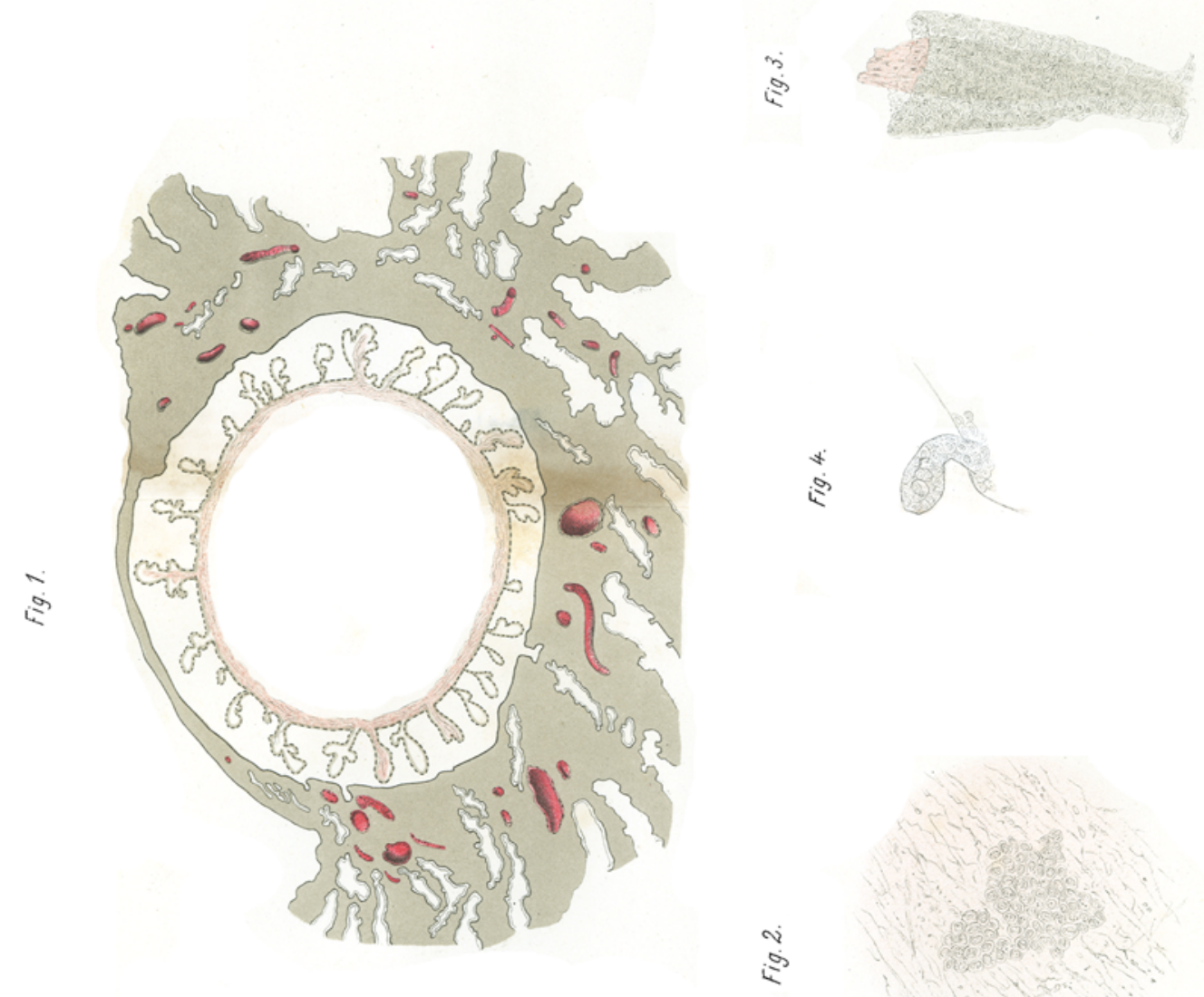